\title{
CD56 and CD11b Positivity with Low Smac/DIABLO Expression as Predictors of Chemoresistance in Acute Myeloid Leukaemia: Flow Cytometric Analysis
}

\author{
Abeer Ibrahim ${ }^{1}$, Asmaa M Zahran², Sanaa Shaker Aly ${ }^{3}$,Ahmed Refaat ${ }^{1}$, \\ Mohammed H Hassan ${ }^{4 *}$
}

\begin{abstract}
Background: Resistance to chemotherapy is a major obstacle to curing acute myeloid leukaemia (AML), and several antigens are claimed to play primary roles in this resistance. Purpose: The aim of this study was to evaluate the roles of CD56, CD11b and Smac/DIABLO gene expression levels as prognostic markers of the clinical outcome, response to chemotherapy and survival of AML patients. Materials and Methods: A cross-sectional observational study was conducted on 60 naïve-AML patients who received induction therapy with mitoxantrone and cytarabine combined with a high dose of cytarabine. The CD56,CD11b and Smac/DIABLO expression levels were assessed using flow cytometry at diagnosis and were analysed for correlation with the possible associated risk factors, response to chemotherapy, and median duration of disease-free survival (DFS) and overall survival (OS). Results: The overall results revealed that AML patients who exhibited positive expression for CD56 and CD11b had short median durations of DFS and OS.(P $=0.019,0.006,0.029$ and 0.024, respectively). Additionally, low Smac/DIABLO expression had a negative impact on treatment outcome in terms of CR rate $(p=0.012)$ and reduced DFS $(p=0.000)$ and $\mathrm{OS}(\mathrm{p}=0.000)$ values. Conclusions: CD56 and CD11b positivity and low Smac/DIABLO expression are important predictive factors for the occurrence of chemoresistance, in addition to other risk factors, among AML patients.
\end{abstract}

Keywords: Chemoresistance- CD56- CD11b- Smac/DIABLO- AML

Asian Pac J Cancer Prev, 19 (11), 3187-3192

\section{Introduction}

Resistance to chemotherapy is a main obstacle to curing acute myeloid leukaemia (AML). Leukaemic cells may exhibit either resistance due to their inherited genotype, or resistance acquired after sub-lethal exposure to anticancer drugs, which frequently occurs in the clinical management of leukaemia. Inherited or acquired genomic alterations lead to the synthesis of gene products responsible for reduced drug sensitivity (Tsuruo, 1988; Gottesman and Pastan,1988).

Among these gene products, the CD56 antigen, an isoform of the neural adhesion molecule on leukaemic blasts, has been proven by some studies to be an independent adverse prognostic factor for the achievement and duration of complete remission (CR), in even good-risk patients (Baer et al., 1997; Raspadori et al., 2001; Di Bona et al., 2002).

Another antigen that has been proven to play a role in resistance to therapy is $\mathrm{CD} 11 \mathrm{~b}$, which is reported to be bound by malignant cells to limit antitumour immunity and promote malignant expansion or refractoriness to treatment (Shojaei et al., 2007; De Veirman et al., 2014; Younos et al., 2015). CD11b is a type of cell surface receptor that is selectively expressed on leukocytes. This protein has several names such as integrin alpha M (ITGAM), complement component 3 receptor alpha chain (CR3a), and macrophage receptor 1 alpha subunit (MAC1a) (Arnaout et al., 1988). This protein can contribute to cell activation, chemotaxis, cytotoxicity, and phagocytosis and controls the interaction of leukaemic cells with the microenvironment via binding to ligands (Fan et al.,1991; Coombe et al.,1994). Therefore, CD11b is thought play an important role in the regulation of the biology of malignant AML cells and the expression level of CD11b may affect the prognosis of AML patients.

Second mitochondria-derived activator of caspases (Smac/DIABLO), a mitochondria-derived proapoptotic protein, enhances the apoptosis by antagonizing the caspase inhibitory effect of inhibitors of apoptosis proteins (IAPs), thereby enhancing apoptosis (Du et al., 2000).

Smac/DIABLO is synthesized in the cytoplasm

${ }^{1}$ Department of Medical Oncology and Haematological Malignancies, ${ }^{2}$ Department of Clinical Pathology, South Egypt Cancer Institute, Assiut University, ${ }^{3}$ Department of Clinical Pathology, ${ }^{4}$ Department of Medical Biochemistry Faculty of Medicine, South Valley University, Qena, Egypt. *For Correspondence: Mohammedhosnyhassaan@yahoo.com 
and imported to the inter-membranous space of mitochondria and was found to play a significant role in the sensitization of cancer cells to various chemotherapeutic regimens (Pluta et al., 2010). Smac/DIABLO is important in the determination of the sensitivity of cancer cells to apoptotic death induced by various stimuli including chemotherapy. Therefore, in the present study, we used flow cytometry to evaluate the role of the immunophenotypic expressions of CD56, CD11b and Smac/DIABLO on clinical outcome and chemotherapeutic response, and the possibility of using these proteins as significant prognostic markers in AML patients.

\section{Materials and Methods}

\section{Study design and participants}

A cross-sectional observational study was carried out on sixty naïve-adult AML patients, who were treated and followed up, within January 2011 and December 2015, respectively. The patients were recruited from the Medical Oncology and Haematological Malignancies Department, South Egypt Cancer Institute, Assiut University, in collaboration with the Clinical Pathology and Medical Biochemistry Departments, South Valley University, Qena, Egypt. The study was approved by the local institutional review board, in accordance with the ethical standards of the responsible committee on human experimentation and with the Declarations of Helsinki (WHO, 2001). Written informed consent was obtained from every patient included in the study after complete explanation of the purposes of the study and the procedures involved. Patients with acute promyelocytic leukaemia were excluded from the study because they were subjected to different treatments. All patients were subjected to thorough history-taking and physical and clinical examination.

\section{Therapeutic protocol}

The treatment used in this study followed the local institutional protocol, which consisted of induction chemotherapy with a 7-day continuous infusion of cytosine-arabinoside $\left(200 \mathrm{mg} / \mathrm{m}^{2}\right)$ and administration of mitoxantrone $\left(12 \mathrm{mg} / \mathrm{m}^{2}\right.$, in 1 hour $)$ for 3 days. Post-induction therapy included the administration of a high dose of cytosine-arabinoside $\left(3,000 \mathrm{mg} / \mathrm{m}^{2}\right.$, twice daily; days 1, 3, and 5) and additional course; patients $>65$ years old received lower doses of the drugs.

\section{Treatment outcomes}

Treatment outcomes were categorized (Suliman et al., 2013), as follows: CR (cellular marrow with less than $5 \%$ of blast cells, a neutrophil count $\geq 1.0 \times 10^{9} / \mathrm{L}$, platelet count $\geq 100 \times 10^{9} / \mathrm{L}$ and no evidence of extramedullary leukaemia); primary resistance (cellular marrow with $>5 \%$ blast cells or evidence of leukaemia in other sites); early relapse (relapse within 6 months after achieving remission). Primary resistance and early relapse accounted for all the early failures. The overall survival (OS) was calculated from the date of diagnosis to the date of death, and the duration of disease-free survival (DFS) was measured from the date of CR until the date of relapse or death, regardless of the cause of death (Cheson et al., 2003).

\section{Laboratory workup}

Bone marrow samples were collected at the time of diagnosis in EDTA tubes for flow cytometric immunophenotyping. The diagnosis was based on clinical and standard morphologic, cytochemical, immunophenotypic, and cytogenetic criteria.

\section{A) Routine laboratory assays included the following}

1. Complete blood pictures were obtained using Cell Dyn 3500 automated cell counter (Abbott Diagnostic, Germany), and peripheral smear examinations were performed manually.

2. Bone marrow examination and cytochemical tests, such as myeloperoxidase, esterases and Periodic acidSchiff (PAS) tests were routinely performed.

3. Immunophenotyping of leukaemic cells was performed on the bone marrow samples using flow cytometry for assessment of a routine panel of monoclonal antibodies (used in diagnosis of AML) against CD34. CD13, CD33, CD117, CD15, intracellular myeloperoxidase, CD14, HLA-DR, CD41, CD61, and glycophorin A. All monoclonal antibodies were purchased from Becton Dickinson (BD) Bioscience, CA, USA.

4. Molecular genetic studies were performed to detect the presence of $\mathrm{t}(15 ; 17)$ (q22;q12) to exclude M3. The $\mathrm{t}(8 ; 21)$, inv $(16)$ and $\mathrm{t}(16 ; 16)$ abnormalities were routinely assessed and defined as favourable prognostic abnormalities, while the $\mathrm{t}(9 ; 22)$ and myeloid/lymphoid leukemia (MLL) gene abnormalities were defined as unfavourable. The FMS-like tyrosine kinase 3 (FLT3) mutation was assessed by routine molecular analysis for all patients. The data regarding the genetic studies were obtained from the patients' files.

\section{B) Specific laboratory assays included the following:}

1. Flow cytometric detection of CD56 and CD11b expression on the blast cells

Fifty microliters of bone marrow sample was incubated with $5 \mu$ of FITC- anti- CD56 and PE- anti CD11b (all from BD Biosciences, CA, USA) for $20 \mathrm{~min}$. at room temperature in the dark. Following incubation, red blood cell (RBC) lysis and washing with PBS were performed. After washing, the cells were resuspended in PBS, and analysed by flow cytometry on a FACS Calibur instrument with Cell Quest software (BD Biosciences, USA). An isotype-matched IgG negative control was used with each sample. Forward and side scatter histograms were used to define the blast cell population. The blast cells were then gated for further analysis of the CD56 and CD11b. CD56 and CD11b expression was considered to be positive when at least $20 \%$ of the blasts expressed the marker.

\section{Flow cytometric detection of the expression of the Smac/DIABLO protein}

An anti-SMAC mouse monoclonal antibody for the human SMAC protein [Anti-Smac/DIABLO antibody [Y12] (Abcam 32023)] raised against a synthetic 
peptide corresponding to $\mathrm{C}$ - terminal residues human Smac/DIABLO) and anti-mouse IgG-FITC were used for detection of the Smac protein (BD Biosciences, San Jose, California USA). Fifty microliters of bone marrow sample was incubated with $5 \mu$ l of primary antibody (Anti-Smac/Diablo) in the dark at $4{ }^{\circ} \mathrm{C}$. After incubation, RBCs were lysed and washed with PBS. The cells were fixed with fixing solution and incubated for 10 minutes. The cells were then washed with PBS, and permeabilization solution and $5 \mu \mathrm{l}$ of secondary anti-mouse IgG-FITC antibody were added and the cells were incubated for 1 hour. The cells were then resuspended in PBS and subjected to flow cytometric analysis on a FACS Calibur instrument with Cell Quest software (BD Biosciences, USA). An IgG isotype-matched negative control was performed with each sample. Forward and side scatter histograms were used to define the blast cell population. The blast cells were then gated for further detection of Smac/DIABLO expression. The expression of Smac/DIABLO is presented as a percentage of leukaemic blasts. High and low expressions were established according to the estimated number of Smac/DIABLO-positive cells in the whole group of patients.

\section{C) Statistics analysis}

Continuous variables were expressed as the mean \pm standard deviation (SD) and $\mathrm{X}^{2}$ test was employed to compare differences between groups. Univariate analyses were used to identify variables affecting response to therapy. DFS and OS values were calculated (Kaplan and Meier, 1958). Differences among groups were compared by the log-rank test. P-values less than 0.05 were considered statistically significant.

\section{Results}

Demographic and clinical data of the included patients with regard to $C D 56, C D 11 b$ and Smac/DIABLO expression (Table 1).

The present study was conducted on sixty AML patients, 39 of whom were males and 21 were females with a median age of 32 years (range 18-61). According to the French-American-British (FAB) criteria, one patient was diagnosed with M0, 3 with M1, 32 with M2, 12 with M4, 11 with M5, one with M6 and none with M7.

CD56 expression was detected in 29/60 (48\%) patients with a mean level of $42 \%$ and CD11b was expressed in $36 / 60(60 \%)$ patients with a mean level of $34 \%$. The median expression of intracellular expression of Smac/DIABLO in the newly diagnosed AML patients was $73.50 \%$ with $30 / 60(50 \%)$ patients exhibiting low expression of Smac/ DIABLO (less than the median).

Regarding CD56, CD11b and Smac/DIABLO expression, there were no statistically significant differences between CD56+, CD56- or CD11b+, CD11b- or high or low Smac/DIABLO in terms of age, gender or FAB class.

CD56, CD11b and Smac/DIABLO expression with regard to other prognostic markers (Table 2).

CD56, CD11b and Smac/DIABLO expression exhibited non-significant relationships with white blood cell (WBC) count, percentage of bone marrow blasts and CD34 expression. There was significantly high FLT3 positivity among CD56+ and CD11b + AML patients, as well as high CD 117 and MLL gene positivity among $\mathrm{CD} 11 \mathrm{~b}+$ patients and patients with low Smac/DIABLO expression.

Association between CD56, CD11b and SMAC/DIABLO expression and treatment outcomes (Table 3).

Response to therapy was observed in all 60 patients who enrolled in the study, 38 out of $60(63 \%)$ of whom achieved CR and 13/60 (22\%) exhibited primary resistance. Two patients $(3 \%)$ died during induction of chemotherapy due to complications associated with treatment, and 7 patients relapsed within 6 months.

CD56, CD11b and Smac/DIABLO expression with regard to the median duration of disease-free survival (DFS) and overall survival (OS) (Figure 1).

The DFS and OS values were significantly lower in the CD56 + and CD11b + groups than in the CD56- and CD11b- groups $(\mathrm{P}=0.019,0.006,0.029$ and 0.024 , respectively). In addition, low Smac/DIABLO expression

Table 1. Demographic and Clinical Data of the Included Patients in Relation to CD56 and CD11b and Smac/DIABLO Expressions

\begin{tabular}{|c|c|c|c|c|c|c|c|c|c|}
\hline Variables $N=60$ & $\begin{array}{l}\text { CD56+ } \\
\mathrm{N}=29\end{array}$ & $\begin{array}{l}\text { CD56- } \\
\mathrm{N}=31\end{array}$ & $*$ p value & $\begin{array}{c}\text { CD11b+ } \\
\mathrm{N}=36\end{array}$ & $\begin{array}{l}\text { CD11b- } \\
\mathrm{N}=24\end{array}$ & $*$ p value & $\begin{array}{c}\text { Smac/DIABLO- } \\
\text { high } \mathrm{N}=30\end{array}$ & $\begin{array}{c}\text { Smac/DIABLO- } \\
\text { low } \mathrm{N}=30\end{array}$ & *p value \\
\hline Age (years), range & $19-55$ & $18-56$ & NS & $18-52$ & $19-56$ & NS & $24-54$ & $25-55$ & NS \\
\hline \multicolumn{10}{|l|}{ Gender } \\
\hline Male & 19 & 20 & NS & 25 & 12 & NS & 18 & 20 & NS \\
\hline Female & 10 & 11 & & 11 & 12 & & 12 & 10 & \\
\hline \multicolumn{10}{|l|}{ FAB Classification } \\
\hline $\mathrm{M} 0(\mathrm{n}=1)$ & 0 & 1 & & 1 & 0 & & 0 & 1 & \\
\hline M1 (n=13) & 1 & 2 & & 2 & 1 & & 1 & 2 & \\
\hline M2 $(n=32)$ & 10 & 22 & NS & 16 & 16 & NS & 14 & 18 & NS \\
\hline M4 (n=12) & 8 & 3 & & 10 & 2 & & 8 & 4 & \\
\hline M5 ( $\mathrm{n}=11)$ & 9 & 2 & & 8 & 3 & & 7 & 5 & \\
\hline M6 (n=1) & 1 & 0 & & 0 & 1 & & 1 & 0 & \\
\hline
\end{tabular}

*Statistically significant at $\mathrm{p} \leq 0.05$; NS, Not statistically significant. 
Table 2. CD56 and CD11b and Smac/DIABLO Expressions in Relation to Other Prognostic Markers

\begin{tabular}{lccccccccc}
\hline Variables N=60 & $\begin{array}{c}\mathrm{CD} 56+ \\
\mathrm{N}=29\end{array}$ & $\begin{array}{c}\mathrm{CD} 56- \\
\mathrm{N}=31\end{array}$ & $\begin{array}{c}\mathrm{p} \\
\text { value }\end{array}$ & $\begin{array}{c}\mathrm{CD} 11 \mathrm{~b}+ \\
\mathrm{N}=36\end{array}$ & $\begin{array}{c}\mathrm{CD} 11 \mathrm{~b}- \\
\mathrm{N}=24\end{array}$ & $\begin{array}{c}\mathrm{p} \\
\text { value }\end{array}$ & $\begin{array}{c}\text { Smac/DIABLO- } \\
\text { high } \\
\mathrm{N}=30\end{array}$ & $\begin{array}{c}\text { Smac/DIABLO- } \\
\text { low } \\
\mathrm{N}=30\end{array}$ & $\begin{array}{c}* \mathrm{p} \\
\text { value }\end{array}$ \\
\hline WBCs $\left(10^{9} / \mathrm{L}\right)$ & $18.75 \pm 1.9$ & $15.89 \pm 2.2$ & $\mathrm{NS}$ & $15.89 \pm 1.9$ & $15.77 \pm 2.2$ & $\mathrm{NS}$ & $12.80 \pm 1.7$ & $16.90 \pm 2$ & $\mathrm{NS}$ \\
Marrow blast $\%$ & $38.98 \pm 2.2$ & $21.98 \pm 2.5$ & $\mathrm{NS}$ & $21.97 \pm 2.5$ & $24.98 \pm 2.2$ & $\mathrm{NS}$ & $21.90 \pm 2.1$ & $22.98 \pm 2.5$ & $\mathrm{NS}$ \\
CD34 \% & $39.91 \pm 1.4$ & $32.57 \pm 3.2$ & $\mathrm{NS}$ & $3.95 \pm 2.8$ & $2.87 \pm 2.2$ & $\mathrm{NS}$ & $29.91 \pm 3.2$ & $2.87 \pm 2.2$ & $\mathrm{NS}$ \\
FLT3 + ve (N=19) & $13 / 29$ & $6 / 31$ & $0.032^{*}$ & $12 / 36$ & $7 / 24$ & $0.030^{*}$ & $6 / 3$ & $13 / 3$ & $\mathrm{NS}$ \\
CD 117+ve (N=24) & $16 / 29$ & $8 / 31$ & $\mathrm{NS}$ & $16 / 36$ & $8 / 24$ & $0.04^{*}$ & $5 / 3$ & $19 / 3$ & $0.02^{*}$ \\
MLL gene +ve (N=18) & $14 / 29$ & $4 / 3$ & $\mathrm{NS}$ & $13 / 36$ & $5 / 24$ & $0.02^{*}$ & $6 / 3$ & $12 / 3$ & $0.039^{*}$ \\
\hline
\end{tabular}

*Statistically significant at $\mathrm{p} \leq 0.05$; NS, Not statistically significant.

Table 3. Association between CD56, CD11b and Smac/DIABLO Expression and Response of Patients to Chemotherapy

\begin{tabular}{|c|c|c|c|c|}
\hline Variables & Complete remission $\mathrm{N}=38$ & Primary resistance $\mathrm{N}=13$ & Early relapse $\mathrm{N}=7$ & *p value \\
\hline \multicolumn{5}{|l|}{ CD11b } \\
\hline$+\mathrm{ve}$ & 16 & 10 & 5 & $0.046^{*}$ \\
\hline -ve & 22 & 3 & 2 & \\
\hline \multicolumn{5}{|l|}{ CD56 } \\
\hline +ve & 15 & 9 & 5 & $0.035^{*}$ \\
\hline -ve & 23 & 4 & 2 & \\
\hline \multicolumn{5}{|c|}{ Smac/DIABLO } \\
\hline High & 24 & 2 & 1 & $0.001 *$ \\
\hline low & 14 & 11 & 6 & \\
\hline
\end{tabular}

*Statistically significant at $\mathrm{p} \leq 0.05$; NS, Not statistically significant.

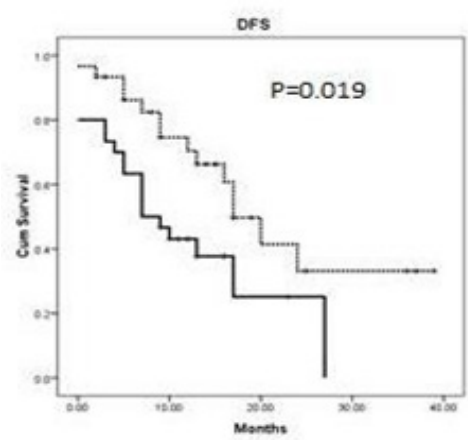

A
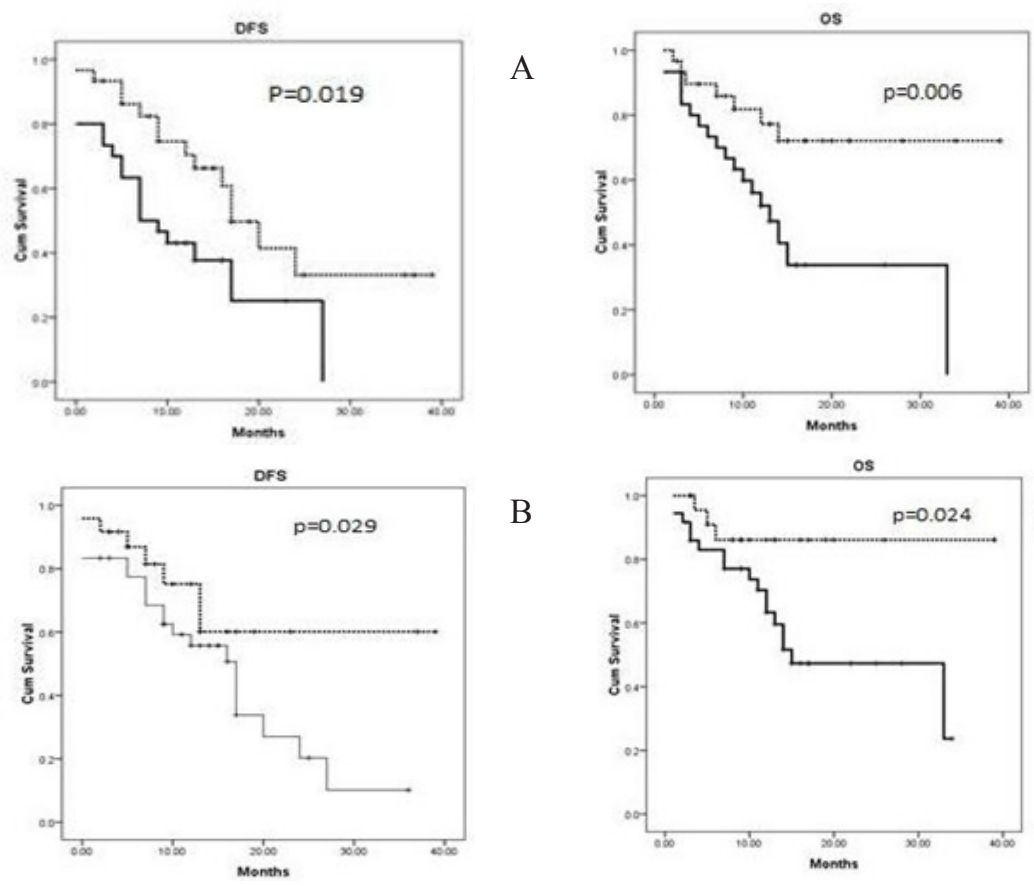

B
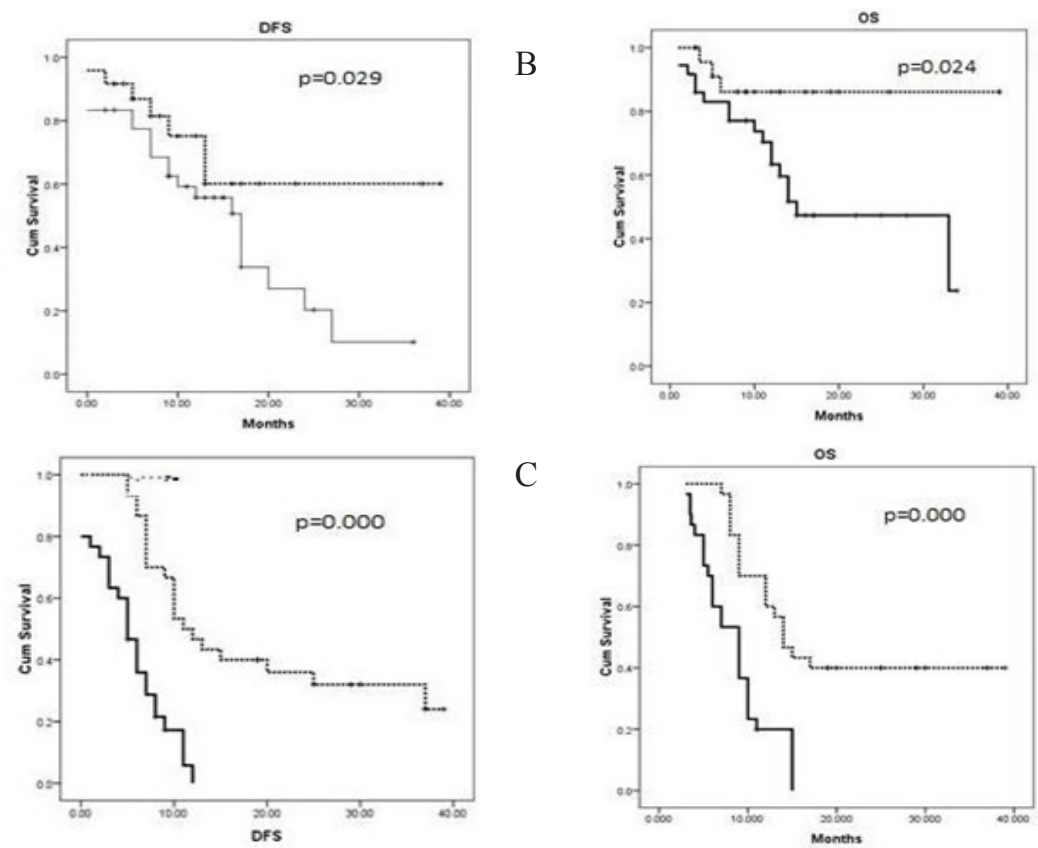

C

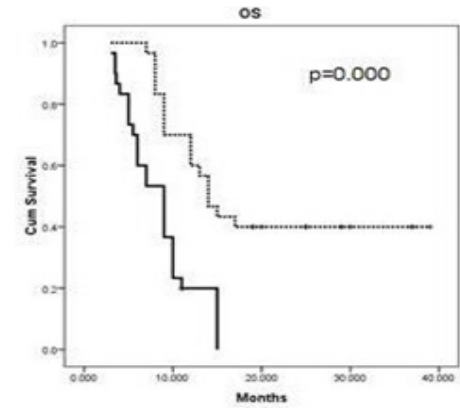

Figure 1. The Kaplan-Meier Curves of CD56 (A), CD11b (B) and Smac/DIABLO (C) Expressions with the Median Duration of Disease-free Survival (DFS) and Overall Survival (OS). 
was associated with poor response to therapy and low, DFS $(\mathrm{P}=0.000)$ and $\mathrm{OS}(\mathrm{P}=0.000)$ values.

There was significant positive correlation between CD56 and CD11b expression ( $r=0.3, p=0.04)$; however, there was no significant correlation between low expression of Smac /DIABLO and positive expression of either CD59 or CD11b ( $\mathrm{p}=0.542$ and 0.93 , respectively).

\section{Discussion}

AML is a common adult leukaemia that has several subgroups with variable clinical features, responses to therapy and prognoses (Siegel et al., 2014; Haferlach, 2008). FCI has an established diagnostic role in AML (Haferlach et al., 2007), but the prognostic significance of this method remains controversial (Webber et al., 2008). The clinical significance of CD11b and CD56 antigen expression as unfavourable prognostic factors for AML has not been elucidated. In addition, literature reviews regarding the evaluation of proapoptotic Smac/DIABLO expression in AML patients are few.

The findings of the present study revealed frequent high expression of CD11b, followed by Smac/DIABLO, and CD56, in approximately half of the included AML patients without significant differences in age, sex or FAB subtype. These findings were consistent with those of many studies (Xu et al., 2015; Pluta et al., 2010; Suliman et al., 2013; Ono et al., 2014).

Cytogenetic parameters are among the most important prognostic parameters for AML, and are used in clinical practice to stratify AML patients and guide treatment (Orend et al., 2013; Bochtler et al., 2013) Our findings showed non-significant associations between CD11b, CD56 and Smac/DIABLO expression with WBC counts and marrow blast or CD $34 \%$, with a significant association observed between CD56+ and CD11b+ with the unfavourable cytogenetic marker FLT3. Additionally, there were significant associations between $\mathrm{CD} 11 \mathrm{~b}+$ and low Smac/DIABLO expression with both the MLL gene and the unfavourable cytogenetic marker CD117. These findings were consistent with the findings of Ciolli et al., (2001), Di Bona et al., (2002), Suliman et al., (2013), Junca et al., (2014) and Xu et al., (2015).

$\mathrm{CD} 11 \mathrm{~b}$ is reported to be involved in restraining antitumour immunity and promoting the expansion and drug resistance of haematological malignant cells (Ahn et al., 2010; Younos et al., 2015). Recently, many investigators have suggested that the expression of the CD56 antigen, a neural adhesion factor, is associated with high relapse rate and poor outcome in acute leukaemia (Ito et al., 2004; Montesinos et al., 2011). Although the actual mechanism via which CD56 in reduces the sensitivity to chemotherapy is unknown, it has been suggested that CD56 is associated with the overexpression of P-glycoprotein (PGP), resulting in a reduction of intracellular drug concentrations (Raspadori et al., 2002). Regarding the treatment outcome and the studied markers, a significant association was observed for primary resistance and early relapse with CD56 and CD11 positivity among the included AML patients. Moreover, our results showed a significant association between CD56 and CD11b expression with low OS and DFS values, which was consistent with the results of Amirghofran et al., (2001) and Xu et al., (2009).

Research on the various pathways implicated in the escape from apoptosis (programmed cell death) is essential and aids the management of AML. Imbalance between cell survival and death resulting from the ability of cancer cells to escape apoptosis, plays an important role in the development of AML, therapeutic resistance and cancer progression (Zhivotovsky and Orrenius, 2006; Zhivotovsky and Orrenius, 2010; Pan et al., 2016; Apidi et al., 2018). Although, in the present study, high and low Smac/DIABLO protein expression appeared to be equally distributed among the included AML patients, subsequent data analysis revealed that low expression levels of Smac/DIABLO protein are associated with reduced sensitivity to standard chemotherapy in the form of primary resistance and early relapse, with low DFS and OS values observed in AML patients. This finding was comparable to the data reported by Pluta et al., (2010) and Pluta et al., (2015), who reported that the decreased expression of Smac/DIABLO and unfavourable cytogenetic parameters were associated with treatment failure. The authors also observed that high Smac/DIABLO gene expression is an independent prognostic factor for high CR rates and high OS values as cells with high Smac/DIABLO expression had low apoptotic resistance, leading to improved responsiveness to treatment and reduced susceptibility to cancer progression (Suliman et al., 2013).

To the best of our knowledge, there are no published studies regarding the correlation of $\mathrm{CD} 56+$ or, $\mathrm{CD} 11 \mathrm{~B}+$ with Smac/DIABLO expression. The present study revealed a significant positive correlation between CD56+, and CD11B+, with non-significant correlations observed between the studied markers, which highlights the independent roles of these proteins and confirms the heterogeneous nature of AML.

In conclusion, the present study confirms that CD56 and CD11b positivity with low expression of Smac/DIABLO could be considered as a useful indicator of chemoresistance and a poor prognostic marker in AML patients, along with other known cytogenetic prognostic markers. In terms of clinical significance, this expression pattern might be helpful in drug targeting, drug design and therapy and could aid the decision making process for different AML treatment strategies.

\section{Disclosure}

The authors declare that there are no conflicts of interest associated with this work.

\section{Funding}

No funding was provided by any institution for this study; this study was funded by the authors themselves.

\section{Acknowledgments}

We acknowledge the participation of all subjects included in the present study. 


\section{References}

Apidi E, Taib WRW, Hassan R, et al (2018). A review on effect of genetic features on treatment responses in acute myeloid leukemia. Metagene, 18, 31-8.

Arnaout MA, Gupta SK, Pierce MW (1988). Amino acid sequence of the alpha subunit of human leukocyte adhesion receptor Mo1 (complement receptor type 3). J Cell Biol, 106, 2153-8.

Baer MR, Stewart CC, Lawrence D (1997).Expression of the neural cell adhesion molecule CD56 is associated with short remission duration and survival in acute myeloid leukemia with $\mathrm{t}(8 ; 21)$ (q22;q22). Blood, 90, 1643-8.

Bulletin of the World Health Organization (2001). Declaration of Helsinki ,79, pp 373-4.

Cheson BD, Bennett JM, Kopecky KJ, et al (2003). Revised recommendations of the International Working Group for Diagnosis, Standardization of Response Criteria, Treatment Outcomes, and Reporting Standards for Therapeutic Trials in Acute Myeloid Leukemia. J Clin Oncol, 21, 4642-9.

Chung S(2014). Febrile seizures. Korean J Pediatr, 57, 384-95.

Ciolli S, Leoni F, Nozzoli C, et al (2001). Prognostic impact of CD56 in 141 cases of acute myeloid leukemia. Haematologica, 10, 86:a17.

Coombe DR, Watt SM, Parish C (1994). Mac-1 (CD11b/CD18) and CD45 mediate the adhesion ofhematopoietic progenitor cells to stromal cell elements via recognition of stromal heparin sulfate. Blood, 84, 739-52.

De Veirman K, Van Valckenborgh E, Lahmar Q ,et al (2014). Myeloid-derived suppressor cells as therapeutic target in hematological malignancies. Front Oncol, 4, 349.

Di Bona E, Sartori R, Zambello R, et al (2002). Prognostic significance of CD56 antigen expression in acute myeloid leukemia. Haematologica, 87, 250-6.

Du C, Fang M, Li Y, et al (2000).Smac, a mitochondrial protein that promotes cytochrome c-dependent caspase activation by eliminating IAP inhibition. Cell, 102, 33-42.

Fan ST, EdgingtonTS (1991). Coupling of the adhesive receptor $\mathrm{CD} 11 \mathrm{~b} / \mathrm{CD} 18$ to functional enhancement of effector macrophage tissue factor response. J Clin Invest, 87, 50-7.

Gottesman MM, Pastan I (1988). Resistance to multiple chemotherapeutic agents in human cancer cells. Trends Pharmacol Sci, 9, 54-8.

Haferlach T (2008). Molecular genetics pathways as therapeutic targets in acute myeloid leukemia. Hematol Am Soc Hematol Educ Program, 2008, 400-11.

Haferlach T, Bacher U, Kern W, et al (2007).. Diagnostic pathways in acute leukemias: a proposal for a multimodal approach. Ann Hematol, 86, 311-27.

Ito S, Ishida Y, Oyake T, et al (2004). Clinical and biological significance of CD56 antigen expression in acute promyelocytic leukemia. Leuk Lymphoma, 45, 1783-9.

Juncà J, Garcia-Caro M, Granada I, et al (2014). Correlation of CD11b and CD56 expression in adult acute myeloid leukemia with cytogenetic risk groups and prognosis. Ann Hematol, 93, 1483-9.

Kaplan EL, Meier P (1958). Nonparametric estimation from incomplete observations. J Am Stat Assoc, 53, 457-81.

Montesinos P, Rayon C, Vellenga E, et al (2011). Clinical significance of CD56 expression in patients with acute promyelocytic leukemia treated with alltrans retinoic acid and anthracycline-based regimens. Blood, 117, 1799-805.

Ono T, Takeshita A, Kishimoto Y, et al (2014). Expression of CD56 is an unfavorable prognostic factor for acute promyelocytic leukemia with higher initial white blood cell counts. Cancer Sci, 105, 97-104.

Pan ST, Li ZL, He ZX, et al (2016). Molecular mechanisms for tumour resistance to chemotherapy. Clin Exp Pharmacol Physiol, 43, 723-37.

Pluta A, Wrzesien-Kus A, Cebula-Obrzut B, et al (2010). Influence of high expression of SMAC/DIABLO protein on the clinical outcome in acute myeloid leukemia patients. Leuk Res, 34, 1308-13.

Pluta A, Wierzbowska A, Cebula-Obrzut B, et al (2015). Prognostic value of inhibitor of apoptosis protein family expression in patients with acute myeloid leukemia. Leuk Lymphoma, 56, 2529-35.

Pluta A, Wrzesien-Kus A, Cebula-Obrzut B, et al (2014). Influence of high expression of Smac/DIABLO protein on the clinical outcome in acute myeloid leukemia patients. Leuk Res, 34, 1308-13.

Raspadori D, Damiani D, Lenoci M, et al (2001). CD56 antigenic expression in acute myeloid leukemia identifies patients with poor clinical prognosis. Leukemia, 15, 1161-4.

Raspadori D, Damiani D, Michieli M, et al (2002). CD56 and PGP expression in acute myeloid leukemia: impact on clinical outcome. Haematologica, 87, 1135-40.

Shojaei F, Wu X, Malik AK, et al (2007). Tumor refractoriness to anti- VEGF treatment is mediated by $\mathrm{CD} 11 \mathrm{~b}+\mathrm{Gr} 1+$ myeloid cells. Nat Bio Technol, 25, 911-20.

Siegel R, Ma J, Zou Z, et al (2014). Cancer statistics, 2014. CA Cancer J Clin, 64, 9-29.

Suliman GA, Mabrouk MM, Rabee ES, et al (2013). Smac/DIABLO gene expression in acute myeloid leukemia patients. Egypt Soc Haematol, 38, 80-3.

Tsuruo T (1988). Mechanism of multidrug resistance and implications for therapy. Jpn J Cancer Res, 79, 285-96.

Webber BA, Cushing MM, Li S (2008). Prognostic significance of flow cytometric immunophenotyping in acute myeloid leukemia. Int J Clin Exp Pathol, 1, 124-33.

Xu S, Li X, Zhang J, et al (2015). Prognostic value of CD11b expression level for acute myeloid leukemia patients: A meta-analysis. PLoS One, 10, e0135981.

Younos IH, Abe F, Talmadge JE (2015). Myeloid-derived suppressor cells: their role in the pathophysiology of hematologic malignancies and potential as therapeutic targets. Leuk Lymphoma, 56, 2251-63.

Zhivotovsky B, Orrenius S (2006). Carcinogenesis and apoptosis: paradigms and paradoxes. Carcinogenesis, 27, 1939-45.

Zhivotovsky B, Orrenius S (2010). Cell death mechanisms: cross-talk and role in disease. Exp Cell Res, 316, 1374-83.

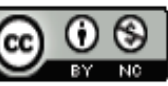

This work is licensed under a Creative Commons AttributionNon Commercial 4.0 International License. 